\title{
The association between idiopathic environmental intolerance and psychological distress, and the influence of social support and recent major life events
}

\author{
Sine Skovbjerg • Alice Rasmussen • Robert Zachariae • \\ Lone Schmidt $\cdot$ Rikke Lund $\cdot$ Jesper Elberling
}

Received: 8 November 2010/ Accepted: 9 February 2011/Published online: 1 March 2011

(C) The Japanese Society for Hygiene 2011

\begin{abstract}
Objectives Idiopathic environmental intolerance (IEI) is a disorder characterized by non-specific symptoms attributed to common airborne chemicals. Increasing evidence points to an association between IEI and symptoms of psychological distress. However, whether other risk factors influence this association has not been clarified. The objective of this study was to examine the association between psychological distress and IEI and to determine whether the association is confounded by social support and major life events.

Methods Data were collected by postal questionnaires; other results from the study have been published previously in this journal. The study included participants from a general population-based study who had reported symptoms of chemical sensitivities $(n=787)$ and two patient groups. The first patient group $(n=101)$ included individuals who had contacted the Danish Research Centre for Chemical Sensitivities, and the second included individuals who had been diagnosed with environmental intolerance
\end{abstract}

S. Skovbjerg $(\varangle) \cdot$ J. Elberling

Department of Dermato-Allergology,

The Danish Research Centre for Chemical Sensitivities,

Copenhagen University Hospital Gentofte,

Ledreborg Alle 40, 2, 2820 Gentofte, Denmark

e-mail: sinsko01@geh.regionh.dk

A. Rasmussen

Psychiatric Centre, Bispebjerg,

University of Copenhagen, Copenhagen, Denmark

R. Zachariae

Psychooncology Research Unit,

Aarhus University Hospital, Aarhus, Denmark

L. Schmidt · R. Lund

Section of Social Medicine, Department of Public Health,

University of Copenhagen, Copenhagen, Denmark
( $n=136)$. Multiple, hierarchical linear regression analyses were conducted with four IEI-related domains, i.e., mucosal and CNS symptoms, chemical intolerances and social consequences, as the dependent variables, and psychological distress, social support and major life events as the independent variables.

Results Our study confirmed positive and statistically significant associations between psychological distress and IEI. The associations remained statistically significant after adjusting for major life events and social support.

Conclusions The results suggest that the association between IEI and psychological distress cannot be explained by known risk factors. More studies, including longitudinal studies, are needed to determine the role of psychological distress in the development and course of IEI.

Keywords Depressive symptoms · Idiopathic environmental intolerance · Psychological distress ·

Social networks · Major life events

$\begin{array}{ll}\text { Abbreviation } \\ \text { CHS } & \text { Chemical Hypersensitivity Scale } \\ \text { CNSS } & \text { CNS Symptom Scale } \\ \text { CSAS } & \text { Consequences for Social Activities Scale } \\ \text { IEI } & \text { Idiopathic environmental intolerance } \\ \text { MUSS } & \text { Mucosal Symptoms Scale } \\ \text { RLE } & \text { Recent Life Events } \\ \text { SCL-92 } & \text { Symptom Checklist } 92\end{array}$

\section{Introduction}

Idiopathic environmental intolerance (IEI) is a disorder characterized by reports of non-specific symptoms from 
various organ systems attributed by the individual to exposure to common airborne chemicals $[1,2]$. In general the reported symptoms are attributed to previous chemical exposures and recur on a subsequent exposure to the same or structurally unrelated chemicals at levels normally considered to be non-toxic [2]. There is currently no internationally accepted diagnostic criteria for IEI [3-5]. Prevalence estimates, point or lifetime, vary widely in population-based studies [6-11], whereas those of physician-diagnosed IEI or reports of disabling consequences in the form of social and occupational disruptions range from 0.5 to $6.3 \%[6,7,10,11]$. Thus, only a subset of individuals who report being sensitive actually consider themselves to be clinically ill or functionally disabled by their symptoms.

Increasing evidence points to an association between IEI and symptoms of psychological distress, i.e., depressive symptoms, negative affect, and anxiety [12-17], and individuals with IEI have been found to have significantly higher scores on measures of psychological distress, such as the Symptom Checklist 92 (SCL-92), than healthy controls [18]. In general, this association is also well established in related disorders, such as fibromyalgia and chronic fatigue syndrome [19-21], but as in IEI, the mechanisms underlying this commonly reported interrelationship has not been clarified. Higher rates of lifetime psychiatric disorders have also been reported [22]. However, not all individuals who report IEI meet the criteria for a current psychiatric disorder [15], and whether psychiatric disorders pre-exist the onset of IEI is not consistent in the literature [22, 23]. Studies aimed at gaining a deeper understanding of the association between IEI and symptoms of psychological distress are needed since psychological distress is likely to add to the level of overall functional disability. Whether factors such as impaired social support or external stressors in terms of major life events, which are known to increase the risk for onset of major depression [24-26], may act as confounders in the association has not been determined.

Social support is one dimension of social relations, a conceptual framework covering two dimensions, i.e., structure and function [27]. The structure refers to both informal and formal interpersonal relations whereas the functional dimension includes the qualitative and behavioural aspects of social relations [27]. Social support can be defined as the level of emotional and instrumental support provided by others [27] and has been identified as a perpetuating factor in the severity of chronic fatigue syndrome [28]. It can be speculated that individuals affected by IEI may experience less social support mainly due to the controversies surrounding the nature of IEI. It may also be hypothesized that symptoms of psychological distress reflect a more severe state of IEI as opposed to less severely affected individuals.

The objectives of the study reported here were to examine whether: (1) self-reported depressive symptoms are associated with a more severe state of IEI among individuals with suspected or diagnosed IEI, (2) the association differs between four IEI-related domains, i.e., mucosal symptoms, central nervous system (CNS) symptoms, symptom-eliciting chemical exposures and social consequences attributed to IEI and (3) the association is confounded by major, recent life events and low perceived social support.

\section{Materials and methods}

\section{Participants}

Results from this study have been published recently in Environmental Health and Preventive Medicine [13, 29]. Therefore, for a more detailed description of the study participants, we refer the reader to our previously published results on the association between perceptual personality characteristics, such as somato-sensory amplification, autonomic perception, absorption and alexithymia, repressive-coping strategies, and IEI [13, 29]. In brief, our conclusions were that somato-sensory amplification and autonomic perception are significantly associated with symptoms attributed to IEI. We found no support for an association between IEI and repressive coping and alexithymia, although there was evidence of a role of one alexithymia domain: difficulties identifying feelings. The association was, however, mediated by negative affectivity, a conclusion which was further supported by findings of relatively strong independent associations between negative affectivity and IEI.

In summary, three groups were invited to participate in the study (1) individuals from the general population ( $n=787$ ), (2) individuals who had contacted the Danish Research Centre because of symptoms attributed to common airborne chemicals $(n=101)$, and (3) patients with physician-diagnosed environmental intolerance $(n=136)$. A total of 1024 women and men were invited to participate. Throughout this article, group 1 is referred to as the population group; groups 2 and 3, which were pooled in order to increase the statistical power, are referred to as the patient group. The overall response rate was $71.5 \%(n=732)$.

\section{Measurements}

A number of self-report measures were included in the study. 


\section{Symptom Checklist 92}

The Symptom checklist 92 (SCL-92) subscale for depression was included as a measure of depressive symptoms or psychological distress. The depression subscale consists of 13 items in which responses are rated on a 5-point Likert scale ranging from not at all to very much. The SCL-92 questionnaire comprises all 92 items from the two other versions of the questionnaire, i.e., the Symptom checklist 90 and the Symptom checklist 90-Revised (SCL-90-R). The SCL-90-R is a revised version of the SCL-90 in which two items on the anxiety subscale have been replaced [30]. The SCL-92 has been validated in a general Danish population, and normative data have been established [30, 31]. The internal validity of the Danish translation of the SCL92 depression subscale has been reported as acceptable (Loevinger coefficient of homogeneity 0.52) [31].

\section{Social support}

Based on the conceptual framework on social relations described by Due and colleagues [27], social support was examined using the following question "will any of the following people help or support you in everyday life, if you need it?" Responses were rated on a 6-point Likert scale ranging from always to never or have none in relation to partner, family, friends, colleagues and neighbours. A high score suggests less perceived social support.

\section{Recent life events}

Recent Life Events (RLE) is a questionnaire that includes a list of potentially stressful major life events, such as disease, loss of a child or partner, unemployment or financial troubles, which may occur in a person's life and which are judged by the respondent as having had a negative impact on his/her quality of life [32]. The score expresses the number of major life events that have occurred within the past year and are rated as having had a negative impact by the respondent [32].

\section{Socio-economic position}

Socio-economic position was measured as occupational social class, and the questionnaire included standardized questions on school education, vocational training and occupation. Data on occupational social class were categorized into a descending scale ranging from occupational social class I-VII. Occupational social class I includes professionals and executives; II, medium-level white-collar employees; III, low-level white-collar employees; IV, skilled workers; V, unskilled and semi-skilled workers. In addition, another two groups were formed, including individuals receiving pension or disability benefits (VI) and students (VII). This approach is in accordance with the standard of the Danish National Institute of Social Research, which is comparable to the British Registrar General's Classification I-V [33]. The occupational social class variable was summarized into three groups in the statistical analysis: (1) professionals and executives and medium-level white-collar employees; (2) low-level whitecollar employees and skilled workers; (3) unskilled and semi-skilled workers, individuals receiving pension or disability benefits and students [33].

\section{Idiopathic environmental intolerance}

Idiopathic environmental intolerance was assessed by questions covering different domains of importance in IEI: (1) number and severity of CNS symptoms, (2) number and severity of mucosal symptoms, (3) number of symptominducing chemicals and (4) social consequences attributed to IEI. Details on the questions included in the questionnaire have been published in Skovbjerg et al. [13, 29].

\section{Statistical analysis}

Principal components analysis

Altogether, 63 IEI items, covering CNS and mucosal symptoms, symptom-eliciting chemicals and consequences in terms of the degree to which reactions had influenced social relations and work, were analysed using principal components analysis (PCA) with Varimax rotation. Details on the statistical analysis are published in Skovbjerg et al. [13, 29]. The results from the PCA were summarized into four IEI scales: (1) the CNS Symptoms Scale (CNSS), which has eight items and total scores ranging from 0 to 8; (2) the Mucosal Symptoms Scale (MUSS), which consists of six items and yields a total score ranging from 0 to 6 ; (3) the Chemical Hypersensitivity Scale (CHS), which has 11 items and yields a total score ranging from 0 to 33 ; (4) the Consequences for Social Activities Scale (CSAS), which has seven items and a total score ranging from 0 to 14 .

Multiple, hierarchical linear regression analyses

Multiple, hierarchical linear regression analyses were performed using the four IEI variables derived from the PCA (CNSS, MUSS, CSAS, CHS) as the dependent variables. The SCL-92 depressive subscale was entered as the independent variable at step one. Social support and the RLE variable were entered at step 2. Age, sex, occupational social class and patient versus population group were entered as control variables at the final and third step of the analysis. 
All data were analysed using SPSS ver. 15.0, and the level of significance was set at $p<0.05$.

\section{Study approval}

The study was approved by the Danish Data Protection Agency. According to Danish legislation, questionnaire studies do not need approval by an ethics committee.

\section{Results}

Group characteristics

The characteristics of the two groups are summarized in Table 1. There were statistically significant differences between the population and the patient group on a number of variables. The patient group was significantly older than the population group and scored significantly higher on the four IEI-related domains (CNSS, MUSS, CSAS, CHS). The patient group also scored higher on the SCL-92 subscale for depression and reported experiencing less social support, whereas scores on the RLE did not differ significantly between the two groups. The two groups also differed on the distribution of occupational social class (Table 1).
Non-respondents

Comparison of respondents and non-respondents in the population group (group 1) showed that respondents to our questionnaire were significantly older, 46.5 years [standard deviation (SD) 12.4] versus 42.9 years (SD 12. 8, $p<0.001$ ), and were more likely to be females [odds ratio (OR) $1.2, p=0.08$ ]. No difference in mean age was seen between respondents and non-respondents in the patient group (group 2) [52.7 years (SD 10.9) and 51.8 years (SD 12.1 ), respectively; $p=0.56]$. Correspondingly, no difference was found for the distribution of $\operatorname{sex}(p=0.54)$.

\section{Correlations}

In the analysis of all study participants, significant moderate to high correlations were found between the four dependent variables (CNSS, MUSS, CSAS and CHS). Moderate and significant correlations were found between the SCL-92 depressive subscale and the four dependent IEI-related domains, while small to moderate correlations were found between social support (SS) and the dependent variables and the SCL-92 subscale. A number of small to moderate correlations were also found between the independent variables (the SCL-92 depressive subscale, RLE and social support) and control variables (age and sex) (Table 2).

Table 1 Group characteristics

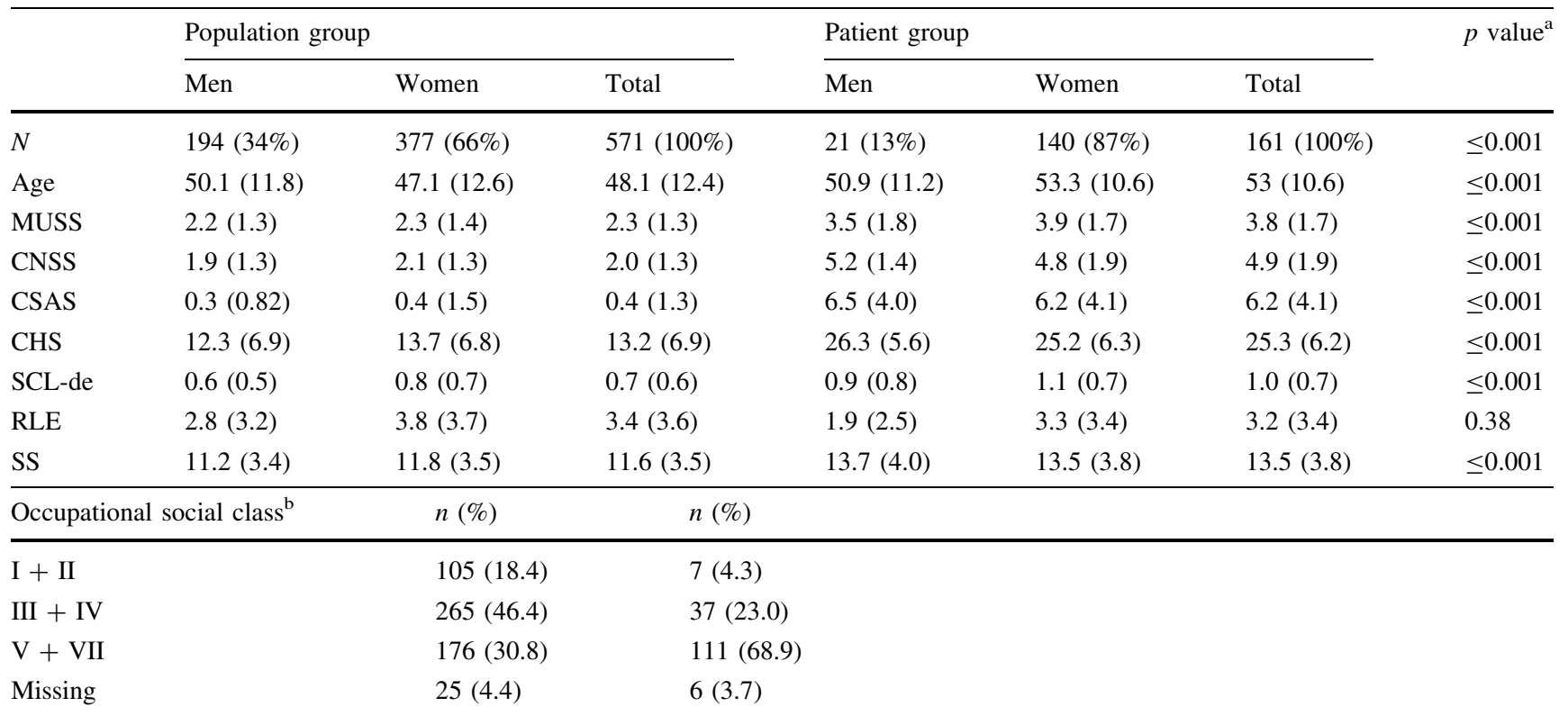

MUSS Mucosal Symptom Scale, CNSS CNS Symptom Scale, CSAS Consequences for Social Activities Scale; CHS Chemical Hypersensitivity Scale, SCL-de SCL-92 subscale for depression, RLE Recent Life Events, SS Social support

${ }^{\mathrm{a}}$ Independent samples $t$ test for equality of means (total) between population and patient sample

b I + II: professionals and executives and medium-level white-collar employees, III + IV: low-level white-collar employees and skilled workers; and V + VII: unskilled and semi-skilled workers, individuals receiving pension or disability benefits and students 
Table 2 Correlations ${ }^{a}$ between the IEI variables, SCL-de, SS, RS, RLE, age, and sex

\begin{tabular}{lllllllrr}
\hline & CNSS & CSAS & CHS & SCL-de & SS & RLE & Age \\
\hline MUSS & $0.60^{* *}$ & $0.41^{* *}$ & $0.47^{* *}$ & $0.21^{* *}$ & $0.15^{* *}$ & $0.15^{* *}$ & $0.09^{*}$ \\
CNSS & - & $0.56^{* *}$ & $0.60^{* *}$ & $0.30^{* *}$ & $0.21^{* *}$ & $0.09^{*}$ & $0.14^{* *}$ \\
CSAS & - & - & $0.56^{* *}$ & $0.25^{* *}$ & $0.25^{* *}$ & 0.01 & 0.06 & $0.15^{* *}$ \\
CHS & - & - & - & $0.29^{* *}$ & $0.18^{* *}$ & $0.08^{*}$ & $0.13^{* *}$ \\
SCL-de & - & - & - & - & $0.31^{* *}$ & $0.37^{* *}$ & -0.04 & $0.18^{* *}$ \\
SS & - & - & - & - & - & $0.14^{* *}$ & 0.05 & $0.17^{* *}$ \\
RLE & - & - & - & - & - & - & $-0.14^{* *}$ \\
Age & - & - & - & - & - & $-0.12^{* *}$ \\
\hline
\end{tabular}

${ }^{a}$ Correlations: *significant at the 0.05 level (2-tailed), **significant at the 0.01 level (2-tailed)

Table 3 Results of a multiple, hierarchical linear regression analysis with the four IEI variables as the dependent variables

\begin{tabular}{|c|c|c|c|c|c|c|c|c|c|}
\hline \multirow[t]{3}{*}{ Step } & \multirow[t]{3}{*}{ Independent/control variables } & \multicolumn{8}{|c|}{ Dependent variables } \\
\hline & & \multicolumn{2}{|l|}{ CNSS } & \multicolumn{2}{|c|}{ MUSS } & \multicolumn{2}{|l|}{ CSAS } & \multicolumn{2}{|l|}{ CHS } \\
\hline & & B & $p$ & B & $p$ & B & $p$ & B & $p$ \\
\hline 1 & SCL-depression & 0.29 & $<0.0001 *$ & 0.21 & $<0.0001 *$ & 0.25 & $<0.0001 *$ & 0.29 & $<0.0001^{*}$ \\
\hline \multirow[t]{3}{*}{2} & SCL-depression & 0.27 & $<0.0001 *$ & 0.15 & $0.0003 *$ & 0.23 & $<0.0001 *$ & 0.26 & $<0.0001^{*}$ \\
\hline & Recent life events & -0.03 & 0.53 & 0.06 & 0.11 & -0.11 & $0.004 *$ & -0.02 & 0.69 \\
\hline & Social support & 0.13 & $\leq 0.001 *$ & 0.11 & $0.006^{*}$ & 0.19 & $<0.0001 *$ & 0.11 & $0.003 *$ \\
\hline \multirow[t]{8}{*}{3} & SCL-depression & 0.15 & $<0.0001 *$ & 0.06 & 0.12 & 0.09 & $\leq 0.001^{*}$ & 0.16 & $<0.0001^{*}$ \\
\hline & Recent life events & 0.06 & 0.07 & 0.13 & $0.0004 *$ & -0.01 & 0.73 & 0.08 & $0.01 *$ \\
\hline & Social support & 0.01 & 0.88 & 0.01 & 0.72 & 0.04 & 0.13 & -0.01 & 0.68 \\
\hline & Occupational social class & 0.11 & $\leq 0.001^{*}$ & 0.12 & $\leq 0.001 *$ & 0.07 & $0.007 *$ & 0.09 & $0.003 *$ \\
\hline & Age & -0.05 & 0.11 & 0.04 & 0.25 & 0.02 & 0.34 & 0.17 & $<0.0001^{*}$ \\
\hline & Sex & -0.01 & 0.65 & 0.04 & 0.26 & -0.01 & 0.75 & 0.05 & 0.11 \\
\hline & Group & 0.58 & $<0.0001 *$ & 0.37 & $<0.0001^{*}$ & 0.70 & $<0.0001 *$ & 0.50 & $<0.0001^{*}$ \\
\hline & Total adjusted $R^{2}$ & 0.44 & & 0.23 & & 0.59 & & 0.43 & \\
\hline
\end{tabular}

$* p<0.05$

Multiple, hierarchical linear regression analysis

Results of the multiple, hierarchical linear regression analyses are shown in Table 3. Statistically significant associations were found between the four IEI domains and the SCL-92 depression subscale at the first step of the analysis. The inclusion of RLE and SS in the model did not change the significant associations. At the third and final step of the analysis, statistical significant associations were seen between the depression subscale and three of the IEI variables (CNSS, CSAS, CHS) after adjusting for occupational social class, age, sex and group [group 1 (population) and group 2 and 3 (patient)]. For the fourth IEI variable, MUSS, significant associations was seen with RLE. The control variables occupational social class and group were significantly associated with all four dependent variables.

\section{Discussion}

The objectives of this study were to investigate the associations between symptoms of depression and four different IEI-related domains and to determine whether low social support and recent major life events would confound the associations.

Overall, our results confirm a positive association between symptoms of depression and IEI, which has also been reported in more studies [15, 17]. They also show strong, statistically significant associations between depressive symptoms and four IEI-related domains, i.e., CNS and mucosal symptoms, symptom-inducing chemicals and social consequences. The associations remained statistically significant after entering the variables recent major life events and social support into the linear regression model. Entering the variables occupational 
social class and group (population and patient group) at the final step of the analysis negatively influenced the strength of the associations. However, the results nevertheless remained strongly statistically significant for three of the IEI-related domains (CNSS, CSAS, CHS). Due to the cross-sectional study design, it was not possible to determine cause and effect relationships, but our results do suggest that the association between depressive symptoms and IEI cannot be explained by the known risk factors included in this study and that the strength of the association increases with more severe states of IEI. The patient group reported significantly less perceived social support and more depressive symptoms, which could be addressed in more detail in future studies. In terms of interpreting the differences in the distribution in occupational social class between the population and patient groups, the significant differences in age may explain why more patients were categorized as class three, which contains, among others, individuals receiving pension.

An association between symptoms of psychological distress, psychiatric co-morbidities and unexplained physical symptoms is in general well established. Henningsen et al. [20] systematically reviewed 244 studies reporting an association between medically unexplained physical symptoms, generalized anxiety disorders and major depression. Increased rates of major depression and anxiety disorders were seen in patients with functional somatic disorders when compared to either healthy controls or patients with other phenomenologically similar medical diseases, such as rheumatoid arthritis [20]. These findings suggest that major depression and anxiety disorders are common and accompanying features of such disorders and do not favour the assumption that they are primarily psychological reactions to pain or other bodily symptoms [20]. Major depression also co-occurs in individuals with asthma at higher rates than in the general population [34], and atopic illness in childhood is associated with an increased risk of major depression later in life independent of socioeconomic status [34, 35]. Examining possible pathways for the association between asthma and major depression, Van Lieshout et al. [34] concluded that the increased rate of major depression in patients with asthma could be explained by shared pathophysiological factors. This conclusion is supported by results from a twin study by Wamboldt et al. [36], who reported that the association between atopy and symptoms of depression is primarily due to additive genetic influences. The co-morbidity between functional somatic disorders has also been examined in a twin study by Kato et al. [37]. Their data suggested that these disorders share psychobiological pathways, such as responses to stress or regulatory mechanisms in the brain, in part with major depression and generalized anxiety disorders, which may offer some explanation to the often reported associations between such disorders [37]. Whether symptoms of psychological distress and psychiatric co-morbidities are part of the aetiology in IEI or merely act as amplifying factors in some individuals can only be speculated at this point. The controversies surrounding medically unexplained symptoms and the lack of diagnostic possibilities regarding these patients are other questions worth considering when possible sources of distress are evaluated. Since it is likely that the aetiology is multi-factorial, and as in functional disorders [38], it can be argued that the complexities of IEI should be studied from a bio-psycho-social perspective. Such an approach would involve considering (1) the influence of biological factors, such as central sensitization processes, which have been suggested as a mechanism involved in the acquisition and maintenance of IEI [39-41], (2) changes in the reactivity of the hypothalamic-pituitaryadrenal axis [42] and/or psychopathological processes, such as the role of anxiety and depression [17], (3) processes involved in symptom perception and amplification and in emotional regulation $[43,44]$ and (4) socioeconomic factors [7].

Some methodological considerations must be highlighted in relation to the interpretation of our results. We did not include a validated measure of IEI and, therefore, we can not be exactly certain of whether the reported associations do in fact reflect our phenomena of interest. In general, the absence of widely accepted case criteria for establishing the presence and degree of IEI challenges epidemiological and clinical studies in this field. When comparing the population group and the patient group, there were significant differences in relation to the scores of the four IEI domains, possibly suggesting that the individuals in the population group are either less affected by their intolerance reactions or are a more heterogeneous group. We approached this issue by conducting a PCA including 63 items by which we identified four IEI domains, which were subsequently included in the statistical analyses. Furthermore, our results indicate that the association with psychological distress is independent of group affiliation, which supports our primary findings. The inclusion of a control group could have strengthened our study design by supplying information on the influence of psychological distress on the presence of IEI independent of severity. This research question, however, was not the objective of our study.

In conclusion, the results of our study suggest that the association between IEI and psychological distress in terms of symptoms of depression cannot be explained by risk factors known to be involved in major depression, i.e., lack of social support and recent major life events. The differences between the population and the patient group found in the study suggest that psychological distress may be a 
risk factor or part of more severe states of IEI, possibly adding to the level of disability, i.e., social and occupational consequences. There is a need for longitudinal studies in order to determine whether psychological distress may be a risk factor in the course of IEI or whether the associations may be explained by shared central pathways.

Acknowledgements This study was supported by research grants from Aage Bangs Foundation. We thank Søren Vesterhauge DMSc, ENT Consultant, Private Hospital Hamlet, for valuable assistance in enrolling IEI patients in the study.

\section{References}

1. Das-Munshi J, Rubin GJ, Wessely S. Multiple chemical sensitivities: review. Curr Opin Otolaryngol Head Neck Surg. 2007;15:274-80.

2. Graveling RA, Pilkington A, George JP, Butler MP, Tannahill SN. A review of multiple chemical sensitivity. Occup Environ Med. 1999;56:73-85.

3. Cullen MR. The worker with multiple chemical sensitivities: an overview. Occup Med. 1987;2:655-61.

4. Lacour M, Zunder T, Schmidtke K, Vaith P, Scheidt C. Multiple chemical sensitivity syndrome (MCS)-suggestions for an extension of the U.S. MCS-case definition. Int J Hyg Environ Health. 2005;208:141-51.

5. Multiple chemical sensitivity: a 1999 consensus. Arch Environ Health. 1999;54:147-149.

6. Kreutzer R, Neutra RR, Lashuay N. Prevalence of people reporting sensitivities to chemicals in a population-based survey. Am J Epidemiol. 1999;150:1-12.

7. Berg ND, Linneberg A, Dirksen A, Elberling J. Prevalence of self-reported symptoms and consequences related to inhalation of airborne chemicals in a Danish general population. Int Arch Occup Environ Health. 2008;81:881-7.

8. Hausteiner C, Bornschein S, Hansen J, Zilker T, Forstl H. Selfreported chemical sensitivity in Germany: a population-based survey. Int J Hyg Environ Health. 2005;208:271-8.

9. Johansson A, Bramerson A, Millqvist E, Nordin S, Bende M. Prevalence and risk factors for self-reported odour intolerance: the Skovde population-based study. Int Arch Occup Environ Health. 2005;78:559-64.

10. Caress SM, Steinemann AC. A national population study of the prevalence of multiple chemical sensitivity. Arch Environ Health. 2004;59:300-5.

11. Caress SM, Steinemann AC. Prevalence of multiple chemical sensitivities: a population-based study in the southeastern United States. Am J Public Health. 2004;94:746-7.

12. Bailer J, Witthoft M, Bayerl C, Rist F. Syndrome stability and psychological predictors of symptom severity in idiopathic environmental intolerance and somatoform disorders. Psychol Med. 2007:37:271-81.

13. Skovbjerg S, Zachariae R, Rasmussen A, Johansen JD, Elberling J. Attention to bodily sensations and symptom perception in individuals with idiopathic environmental intolerance. Environ Health Prev Med. 2010;15(3):141-50.

14. Osterberg K, Persson R, Karlson B, Carlsson EF, Orbaek P. Personality, mental distress, and subjective health complaints among persons with environmental annoyance. Hum Exp Toxicol. 2007;26:231-41.
15. van Caccappolo-Vliet E, Kelly-McNeil K, Natelson B, Kipen H, Fiedler N. Anxiety sensitivity and depression in multiple chemical sensitivities and asthma. J Occup Environ Med. 2002;44:890-901.

16. Bell IR, Peterson JM, Schwartz GE. Medical histories and psychological profiles of middle-aged women with and without selfreported illness from environmental chemicals. J Clin Psychiatry. 1995;56:151-60.

17. Black DW. The relationship of mental disorders and idiopathic environmental intolerance. Occup Med. 2000;15:557-70.

18. Papo D, Eberlein-König B, Berresheim HW, Huss-Marp J, Grimm V, Ring J, Behrendt H, Winneke G. Chemosensory function and psychological profile in patients with multiple chemical sensitivity: comparison with odor-sensitive and asymptomatic controls. J Psychosom Res. 2006;60:199-209.

19. Gormsen L, Rosenberg R, Bach FW, Jensen TS. Depression, anxiety, health-related quality of life and pain in patients with chronic fibromyalgia and neuropathic pain. Eur $\mathrm{J}$ Pain. 2010;14:127-8.

20. Henningsen P, Zimmermann T, Sattel H. Medically unexplained physical symptoms, anxiety, and depression: a meta-analytic review. Psychosom Med. 2003;65:528-33.

21. Van Houdenhouve B, Kempke S, Luyten P. Psychiatric aspects of chronic fatigue syndrome and fibromyalgia. Curr Psychiatry Rep. 2010;12:208-14

22. Dietel A, Jordan L, Muhlinghaus T, Eikmann TF, Herr CE, Nowak D, Pedrosa GF, Podoll K, Wiesmuller GA, Eis D. Psychiatric disorders of environmental outpatients-results of the standardized psychiatric interview (CIDI) from the German multi-center study on Multiple Chemical Sensitivity (MCS). Psychother Psychosom Med Psychol. 2006;56:162-71.

23. Simon GE. Psychiatric symptoms in multiple chemical sensitivity. Toxicol Ind Health. 1994;10:487-96.

24. Olsen LR, Mortensen EL, Bech P. Prevalence of major depression and stress indicators in the Danish general population. Acta Psychiatr Scand. 2004;109:96-103.

25. Kendler KS, Karkowski LM, Prescott CA. Causal relationship between stressful life events and the onset of major depression. Am J Psychiatry. 1999;156:837-41.

26. Paykel ES. Life events, social support and depression. Acta Psychiatr Scand Suppl. 1994;377:50-8.

27. Due P, Holstein B, Lund R, Modvig J, Avlund K. Social relations: network, support and relational strain. Soc Sci Med. 1999;48:661-73.

28. Prins JB, Bos E, Huibers MJH, Servaes P, van der Werf SP, van der Meer JWM, Bleijenberg G. Social support and the persistence of complaints in chronic fatigue syndrome. Psychother Psychosom. 2004;73:174-82.

29. Skovbjerg S, Zachariae R, Rasmussen A, Johansen JD, Elberling J. Repressive coping and alexithymia in idiopathic environmental intolerance. Environ Health Prev Med. 2010;15:299-310.

30. Olsen LR, Mortensen EL, Bech P. Mental distress in the Danish general population. Acta Psychiatr Scand. 2006;113:477-84.

31. Olsen LR, Mortensen EL, Bech P. The SCL-90 and SCL-90R versions validated by item response models in a Danish community sample. Acta Psychiatr Scand. 2004;110:161-2.

32. Cohen S, Tyrrell DA, Smith AP. Psychological stress and susceptibility to the common cold. N Engl J Med. 1991;325:606-12.

33. Christensen U, Schmidt L, Hougaard CO, Kriegbaum M, Holstein BE. Socioeconomic position and variations in coping strategies in musculoskeletal pain: a cross-sectional study of 1 , 287 40- and 50-year-old men and women. J Rehabil Med. 2006;38:316-21.

34. Van Lieshout RJ, Bienenstock J, MacQueen GM. A review of candidate pathways underlying the association between asthma 
and major depressive disorder. Psychosom Med. 2009;71: 187-95.

35. Cohen P, Pine DS, Must A, Kasen S, Brook J. Prospective associations between somatic illness and mental illness from childhood to adulthood. Am J Epidemiol. 1998;147:232-9.

36. Wambolt MZ, Hewitt JK, Schmitz S, Wambolt FS, Rasanen M, Koskenvuo M, Romanov K, Varjonen J, Kaprio J. Familial association between allergic disorders and depression in adult Finnish twins. Am J Med Genet. 2000;96:146-53.

37. Kato K, Sullivan PF, Evengard B, Pedersen NL. A populationbased twin study of functional somatic syndromes. Psychol Med. 2010;39:497-505.

38. Sharpe M, Mayou R, Walker J. Bodily symptoms: new approaches to classification. J Psychosom Res. 2006;60:353-6.

39. Bell IR, Baldwin CM, Fernandez M, Schwartz GE. Neural sensitization model for multiple chemical sensitivity: overview of theory and empirical evidence. Toxicol Ind Health. 1999;15: 295-304.
40. Yunus MB. Central sensitivity syndromes: a new paradigm and group nosology for fibromyalgia and overlapping conditions, and the related issue of disease versus illness. Semin Arthritis Rheum. 2008;37:339-52.

41. Holst H, Arendt-Nielsen L, Mosbech H, Elberling J. Increased capsaicin-induced secondary hyperalgesia in patients with multiple chemical sensitivity. Clin J Pain. 2010;27:156-62.

42. Van Houdenhouve B, van den Eede F, Luyten P. Does hypothalamic-pituitary-adrenal axis hypofunction in chronic fatigue syndrome reflect a "crash" in the stress system? Med Hypotheses. 2009;72:701-5.

43. Witthöft M, Gerlach AL, Bailer J. Selective attention, memory bias, and symptom perception in idiopathic environmental intolerance and somatoform disorders. J Abnorm Psychol. 2006;115:397-407.

44. Andersson L, Bende M, Millqvist E, Nordin S. Attention bias and sensitization in chemical sensitivity. J Psychosom Res. 2009;66: 407-16. 